\title{
Powerful GeV emission from a $\gamma$-ray-burst shock wave scattering stellar photons
}

\author{
D. Giannios
}

\author{
Max Planck Institute for Astrophysics, Box 1317, 85741 Garching, Germany \\ e-mail: giannios@mpa-garching.mpg.de
}

Received 2 May 2008 / Accepted 22 July 2008

ABSTRACT

\begin{abstract}
The $\gamma$-ray bursts (GRBs) of long duration are very likely to be connected to the death of massive stars. The $\gamma$-ray emission is believed to come from energy released internally in a flow that moves at ultrarelativistic speed. The fast flow drives a shock wave into the external medium leading to the afterglow emission. Most massive stars form in dense clusters, their high luminosity producing a very dense radiation field. Here, I explore the observational consequences of the interaction of the shocked external medium of the burst with the photon field of a nearby $\mathrm{O}$ star. I show that inverse Compton scattering of the stellar photons by electrons heated by the shock leads to powerful $\gamma$-ray emission in the $\sim 1-100 \mathrm{GeV}$ range. This emission appears minutes to hours after the burst and can be easily detected by Cherenkov telescopes and probably with the GLAST satellite. This signal may have already been observed in GRB 940217 and can yield important information about the circumburst environment and the extragalactic background light.

Key words. gamma rays: bursts - radiation mechanisms: general
\end{abstract}

\section{Introduction}

Long-duration $\gamma$-ray bursts (GRBs) are believed to result from the death of Wolf-Rayet stars (MacFadyen \& woosley 1999; Galama et al. 1998; Stanek et al. 2003; Hjorth et al. 2003). Stars rarely form in isolation. This is particularly true for the most massive stars, a large fraction of which form in high-mass clusters of $M_{\mathrm{c}} \gtrsim 10^{4} M_{\odot}$. About $30 \%$ of the known Galactic WolfRayet stars are located in the high-mass clusters Westerlund 1 and the Galactic center clusters (Arches, Quintuplet, and Center; e.g. see Figer 2004; Crowther 2007).

High-mass clusters are characterized by a high concentration of O stars with luminosity about a million times the solar. Such a cluster contains tens to hundreds of O-stars in a typical radius of $3 \times 10^{17}-1 \times 10^{18} \mathrm{~cm}$, so the mean distance between them is $\sim 1-3 \times 10^{17} \mathrm{~cm}$ (Massey \& Hunter 1998; Figer 2004). A large fraction of GRBs is, therefore, expected to take place in a dense environment of luminous stars.

The GRB emission is believed to be the result of internal energy dissipation in a relativistic flow. Additional X-ray $/ \gamma$-ray emission is expected as the result of inverse Compton scattering if the fast flow transverses a dense radiation field. This can take place in the funnel of the collapsing star (Lazzati et al. 2000), during the jet breakout from the collapsar (MacFadyen et al. 2001) or shortly afterwards because of the presence of a stellar companion (Ramirez-Ruiz 2004).

At a much larger distance from the central engine, the ejecta decelerate, interacting with the external medium driving a shock into it. For typical GRB parameters the blast wave remains relativistic at $\sim 0.1 \mathrm{pc}$ distance where it can interact with a nearby $\mathrm{O}$ star. In this paper, I study the $\gamma$-ray emission from this interaction. The $\mathrm{O}$ star provides the UV photons that are upscattered (external Compton) by the electrons accelerated in the forward shock. It is shown that such interaction leads to powerful afterglow emission in the $\sim 1-100 \mathrm{GeV}$ energy range.

\section{The model}

Consider a Wolf-Rayet star exploding at a distance $R_{\mathrm{S}} \gtrsim 10^{17} \mathrm{~cm}$ from an $\mathrm{O}$ star and launching the ultrarelativistic outflow (with bulk $\Gamma \gtrsim 100$ ) that produces a $\gamma$-ray burst. After the burst has taken place, the flow interacts with the external medium driving a relativistic shock into it. The afterglows of GRBs seen in $\mathrm{X}$ rays and lower frequencies are often interpreted as synchrotron emission from this shock (Sari et al. 1998). When the shock approaches the $\mathrm{O}$ star, it sweeps through its dense radiation field. Inverse Compton scattering of this radiation field is an effective way of converting the energy of the shocked medium into high-energy photons.

\subsection{Inverse Compton in the forward shock}

The expected observable flux and energy of the photons of this emission is determined by the energy density of the stellar photon field and the number and energy of the scattering particles in the blast wave.

The shock propagates into a circumburst medium with a complex density profile that is shaped by the interacting winds of the two stars. For typical values of the mass loss rates and wind speeds of the Wolf-Rayet and O stars (Stevens et al. 1992; Chevalier \& Li 2000) and for a distance $R_{\mathrm{S}} \sim$ a few $10^{17} \mathrm{~cm}$, the particle densities of the medium are of order $n \sim 10^{2} \mathrm{~cm}^{-3}$.

The blast wave is still ultrarelativistic at such distances from the source. For a spherical and adiabatic shock, the shocked medium at distance $R_{\mathrm{S}}$ has a Lorentz factor of (Blandford \& McKee 1976)

$\Gamma \simeq\left(\frac{3 E}{4 \pi R_{\mathrm{s}}^{3} n m_{\mathrm{p}} c^{2}}\right)^{1 / 2} \simeq 40 \frac{E_{54}^{1 / 2}}{n_{2}^{1 / 2} R_{17}^{3 / 2}}$,

where $m_{\mathrm{p}}$ and $c$ are the proton mass and the speed of light, and the notation $A=10^{x} A_{x}$ and CGS units are used. Also, $E$ is the 
total energy of the shocked medium (kinetic and thermal). The value $10^{54} \mathrm{erg}$ is typical of the equivalent isotropic energy inferred for GRBs. An estimated fraction of some $10 \%$ of this energy appears as burst emission, but most of the energy (depending on model parameters) converts into an outward propagating shock, and is available for the afterglow process proposed here.

If the energy that is dissipated in the shock were efficiently thermalized and shared among the particles, electrons would be accelerated to a characteristic Lorentz factor $\gamma_{\mathrm{e}} \sim \Gamma m_{\mathrm{p}} / m_{\mathrm{e}}$ in the frame comoving with the shocked medium. However, in the shocked medium under consideration, Coulomb collisions are not an efficient channel for energy exchange between particles and the fate of the energy released in the shock is not clear. I assume, as is customary in afterglow models, that the electrons swept by the shock receive a fraction $\epsilon_{\mathrm{e}} \sim 0.1$ of the dissipated energy. In the frame comoving with the blast wave, the electron distribution is assumed isotropic with a characteristic Lorentz factor (Sari et al. 1998; Wijers \& Galama 1999)

$\gamma_{\mathrm{e}}=\epsilon_{\mathrm{e}} \Gamma m_{\mathrm{p}} / m_{\mathrm{e}} \simeq 7.4 \times 10^{3} \frac{\epsilon_{\mathrm{e},-1} E_{54}^{1 / 2}}{n_{2}^{1 / 2} R_{17}^{3 / 2}}$,

where $\epsilon_{\mathrm{e}}=0.1 \epsilon_{\mathrm{e},-1}$.

\subsubsection{Energy of the inverse compton photons}

In the frame of the collapsing star the energy of the heated electrons is $E_{\mathrm{e}}=4 \Gamma \gamma_{\mathrm{e}} m_{\mathrm{e}} c^{2} / 3$. They transfer part of this energy to the stellar photons through scattering. The scattered photons gain a factor $A \sim 2 \Gamma^{2} \gamma_{\mathrm{e}}^{2}$ with respect to their initial energy $e_{\mathrm{s}}$. Including recoil, the energy gain of a photon is limited to the energy of the scattering electron $E_{\mathrm{e}}$. The characteristic energy of the observed inverse Compton emission for a burst at redshift $z$ is then

$E_{\gamma}=\min \left[\frac{1700}{1+z} \frac{\epsilon_{\mathrm{e},-1}^{2} E_{54}^{2}}{n_{2}^{2} R_{17}^{6}} e_{\mathrm{s}, 1}, \frac{200}{1+z} \frac{\epsilon_{\mathrm{e},-1} E_{54}}{n_{2} R_{17}^{3}}\right] \mathrm{GeV}$,

where $e_{\mathrm{S}}=10 e_{\mathrm{S}, 1} \mathrm{eV}$. The energy of the typical stellar photon is $e_{\mathrm{S}} \simeq 2.7 k_{\mathrm{B}} T_{\mathrm{s}} \simeq 10 \mathrm{eV}$ for a main-sequence $\mathrm{O}$ star of temperature $T_{\mathrm{s}} \sim 45000 \mathrm{~K}$. If the star has evolved into a red supergiant, its temperature is a factor of 10 less, resulting in $e_{\mathrm{s}} \sim 1 \mathrm{eV}$.

\subsubsection{Observed photon fluence}

Since the blast wave moves with a Lorentz factor $\Gamma \gg 1$, the observer sees only emission coming from a cone of opening angle $\sim 1 / \Gamma$ with respect to the line of sight. The flux of high-energy photons expected from inverse Compton scattering depends on the distance of the $\mathrm{O}$ star from the line of sight to the GRB. Let $\theta$ be the angle between the $\mathrm{O}$ star and the observer, as seen from the GRB source, and then distinguish between the on-axis case where $\theta \sim 1 / \Gamma$ and the off-axis case in which $\theta \sim 1$.

The shocked interstellar material forms a thin shell of width $\sim R / \Gamma^{2}$ behind the relativistic blast wave (Blandford \& McKee 1976). When it reaches distance $R_{\mathrm{s}}$, the blast wave has swept through $N_{\mathrm{t}}=4 \pi n R_{\mathrm{s}}^{3} / 3$ electrons out of which $N=N_{\mathrm{t}} / 4 \Gamma^{2}=$ $\pi n R_{\mathrm{s}}^{3} / 3 \Gamma^{2}$ are radiating within the observer's cone. These shockheated electrons have a mean Lorentz factor $4 \gamma_{\mathrm{e}} \Gamma / 3$ (in the frame of the collapsing star). Using the standard expression for Compton scattering by relativistic electrons, the instantaneous energy flux of the up-scattered photons is $F(t)=$ $4 c \sigma_{\mathrm{T}} U_{\text {rad }}\left(4 \gamma_{\mathrm{e}} \Gamma / 3\right)^{2} N / 3$. The $\mathrm{O}$ star's radiation field $U_{\text {rad }}$ can be approximated by its average $\bar{U}_{\text {rad }}$ over the volume $V$ where most scatterings take place (see Fig. 1).

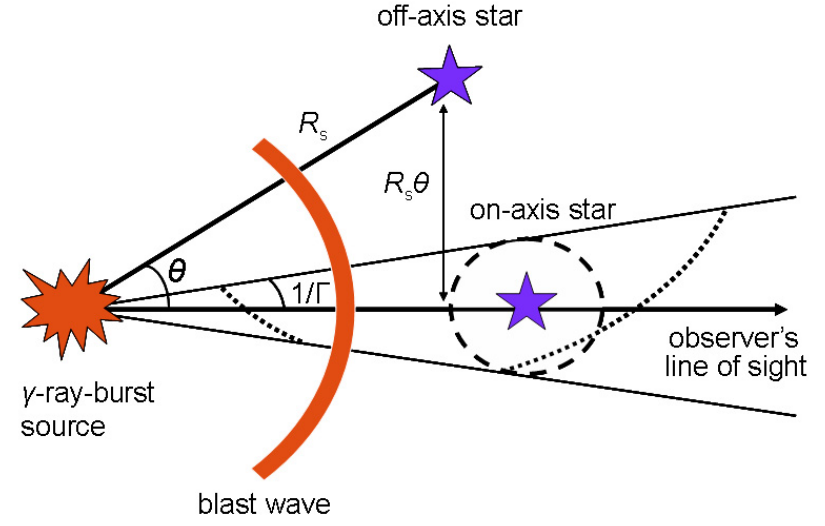

Fig. 1. High-energy photons produced by a relativistic blast wave propagating with a Lorentz factor $\Gamma$ through the radiation field of a luminous O star, located at a distance $R_{\mathrm{s}}$ from the center of the explosion and an angle $\theta$ with respect to the line of sight to the observer. The observer sees only scattered photons that lie within a cone of opening angle $\sim 1 / \Gamma$ (observer's cone). In the on-axis case, the $\mathrm{O}$ star is inside the observer's cone. An off-axis star is located at $\theta \sim 1$. The dashed and dotted curves mark the region where most of the observed scattering events take place for on-axis and off-axis stars respectively.

Integrating the resulting flux in time as the shell crosses through $V$ gives the expression

$F_{\gamma} \sim \frac{\Gamma L_{\mathrm{s}} \sigma_{\mathrm{T}} n R_{\mathrm{s}}^{2}}{5 c d^{2} e_{\mathrm{s}}} \sim 3 \times 10^{-4} \frac{E_{54}^{1 / 2} n_{2}^{1 / 2} L_{39.5} R_{17}^{1 / 2}}{e_{\mathrm{s}, 1} d_{28}^{2}} \mathrm{~cm}^{-2}$,

for the photon fluence for the case of on-axis star. Here, $d=$ $10^{28} d_{28} \mathrm{~cm}$ is the proper distance of the burst that corresponds to a redshift $z=1$ and $L_{\mathrm{s}}=10^{39.5} L_{39.5} \mathrm{erg} / \mathrm{s}$ is the stellar luminosity. The observed photon fluence depends weakly on the various parameters. In the off-axis case the photon fluence is a factor $\sim 1 / \Gamma$ less.

In deriving the last expression, I used that, if the star is on-axis, the stellar radiation in the observer's cone is mainly located within a distance $\sim R_{\mathrm{S}} / \Gamma$ from the $\mathrm{O}$ star. The average energy density of the stellar photon field in this volume is $\bar{U}_{\text {rad }}=\int U_{\text {rad }} \mathrm{d} V / V=3 L_{\mathrm{s}} \Gamma^{2} / 4 \pi c R_{\mathrm{s}}^{2}$. The blast wave crosses this volume at a time $t_{\mathrm{cr}} \sim 2 R_{\mathrm{S}} / \Gamma c$. The total energy of the upscattered photons within the observer's cone is $E_{\mathrm{IC}}^{\text {on }} \sim$ $4 c \sigma_{\mathrm{T}} \bar{U}_{\mathrm{rad}}\left(4 \gamma_{\mathrm{e}} \Gamma / 3\right)^{2} N t_{\mathrm{cr}} / 3 \sim \Gamma \gamma_{\mathrm{e}}^{2} R_{\mathrm{s}}^{2} L_{\mathrm{s}} n \sigma_{\mathrm{T}} / c$. Dividing $E_{\mathrm{IC}}^{\text {on with }}$ the typical energy of the upscattered photons $2 \Gamma^{2} \gamma_{\mathrm{e}}^{2} e_{\mathrm{s}}$ and the surface area $\pi d^{2} / \Gamma^{2}$, one derives the photon fluence on Earth (4). When $1 / \Gamma \ll \theta \ll 1$, the star is located at a distance $R_{\mathrm{s}} \theta$ away from the line of sight. The energy density of stellar photons at this distance is $\bar{U}_{\text {rad }} \simeq L_{\mathrm{S}} / 4 \pi\left(R_{\mathrm{S}} \theta\right)^{2} c$. The blast wave crosses this volume at $t_{\mathrm{cr}} \sim 2 R_{\mathrm{s}} \theta / c$. When extrapolating the previous expressions for $\theta \sim 1$ (off-axis case), one finds $E_{\mathrm{IC}}^{\text {off }} \sim E_{\mathrm{IC}}^{\text {on }} / \Gamma$.

\subsubsection{Klein-Nishina suppression}

Equation (4) holds as long as scattering takes place in the Thomson limit. When the photon energy in the electron rest frame $E^{\prime} \simeq 4 / 3 \Gamma \gamma_{\mathrm{e}} e_{\mathrm{s}}$ exceeds the $m_{\mathrm{e}} c^{2}$, relativistic effects decrease the scattering cross section and consequently the strength of inverse Compton emission. For UV soft photons of $e_{\mathrm{s}} \sim$ $10 \mathrm{eV}$, the Klein-Nishina suppression in the cross section appears for observed $E_{\mathrm{IC}} \gtrsim 25 /(1+z) \mathrm{GeV}$. For IR photons of $e_{\mathrm{s}} \sim 1 \mathrm{eV}$ (expected from a red supergiant), the Klein-Nishina 


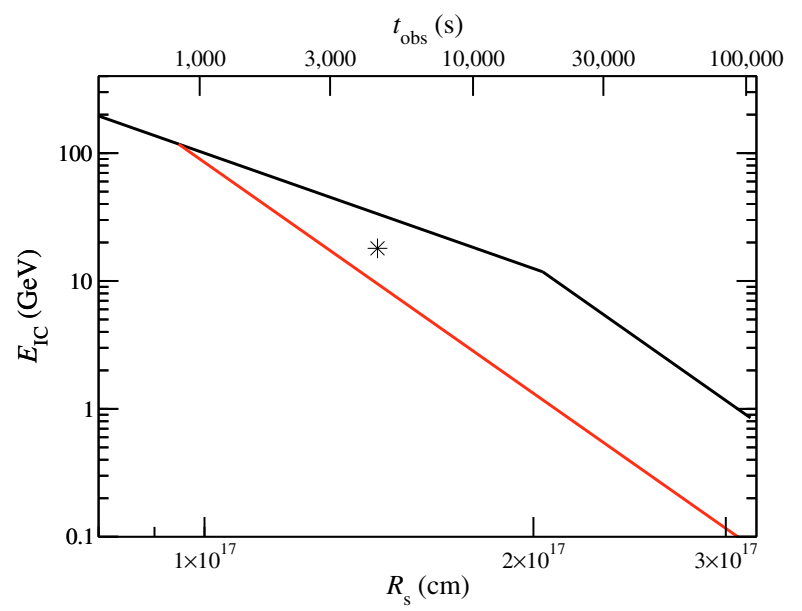

Fig. 2. Observed energy of the scattered photons as a function of distance $R_{\mathrm{S}}$ of the $\mathrm{O}$ star from the center of the explosion. The black curve corresponds to a main-sequence $\mathrm{O}$ star and the red curve to a red supergiant respectively. $t_{\mathrm{obs}}$ stands for the time after the burst that the inverse Compton emission is observed. Photon energy and time are measured in the frame of the observer assuming a burst at $z=1$. The asterisk stands for the $18 \mathrm{GeV}$ photon detected by EGRET $\sim 4500 \mathrm{~s}$ after the burst of the 17 February 1994 assuming a redshift of $z=1$.

suppression appears for $E_{I C} \gtrsim 250 /(1+z) \mathrm{GeV}$. In the extreme relativistic $E^{\prime} \gg m_{\mathrm{e}} c^{2}$ regime, the scattering cross section is

$\sigma=3 \sigma_{\mathrm{T}}(\ln 2 x+1 / 2) / 8 x$,

where $x=E^{\prime} / m_{\mathrm{e}} c^{2}$. The Klein-Nishina suppression is taken into account in the results that follow.

\subsection{Attenuation of the produced $\gamma$-rays}

The $\gamma$-rays can be attenuated by interacting with ambient radiation fields creating electron-positron pairs at the location of production or on the way to Earth. I have verified that the produced $\gamma$-rays are not attenuated significantly by interacting with the radiation field of the $\mathrm{O}$ star, but $\mathrm{I}$ did not include attenuation of the signal from interaction with the extragalactic background light. This becomes significant for observed $\gamma$-rays of energy $E_{\gamma} \gtrsim 100 \mathrm{GeV}$ for a burst that takes place at $z \gtrsim 1$ (e.g. Blanch \& Martinez 2005).

\section{Results: detection prospects}

he resulting energy and strength of the inverse Compton emission are summarized in Figs. 2 and 3. The emission appears in the $\sim 100 \mathrm{GeV}$ and $\sim 1 \mathrm{GeV}$ energy range for a star located at a distance $R_{\mathrm{s}} \sim 10^{17} \mathrm{~cm}$ (hereafter referred to as "close encounter") and $\sim 3 \times 10^{17} \mathrm{~cm}$ (distant encounter) from the center of the explosion, respectively.

The time after the $\gamma$-ray burst at which the observer sees the scattered photons depends on the distance $R_{\mathrm{S}}$ of the star (Sari et al. 1998)

$t_{\mathrm{obs}} \simeq \frac{R_{\mathrm{s}}}{4 \Gamma^{2} c}(1+z) \simeq 520(1+z) \frac{n_{2} R_{17}^{4}}{E_{54}} \mathrm{~s}$

The duration of the emission $\delta t$ is determined by the different travel times of the scattered photons and is about $t_{\mathrm{obs}}$. A close encounter results in emission tens of minutes after the burst while a distant encounter is observed about half a day after the burst.

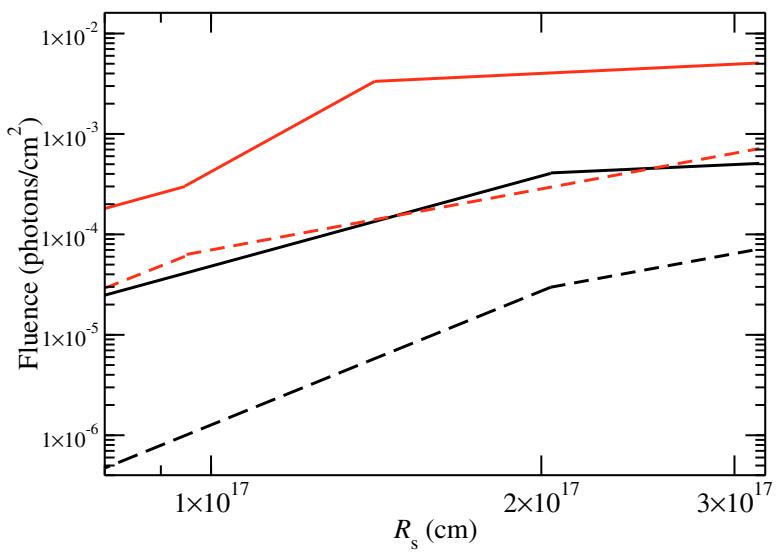

Fig. 3. Observed fluence of scattered stellar photons as a function of distance $R_{\mathrm{s}}$ of the $\mathrm{O}$ star from the center of the explosion. The burst is assumed to take place at redshift $z=1$. The black curves correspond to a main-sequence $\mathrm{O}$ star and the red curves to a red supergiant. The solid curves correspond to the case where the star is located in the line of sight and the dashed curves to an off-axis star.

The observed photon fluence from a burst at $z=1$ is shown in Fig. 3. It ranges from $\sim 5 \times 10^{-7}$ photons $/ \mathrm{cm}^{2}$ to $\sim 5 \times 10^{-3}$ photons $/ \mathrm{cm}^{2}$ for an off-axis main sequence $\mathrm{O}$ star and an on-axis red supergiant, respectively.

A close encounter results in emission within the observed energy range of Cherenkov telescopes, especially that of MAGIC II and HESS II. With MAGIC's effective area of $\sim 3 \times 10^{8} \mathrm{~cm}^{2}$ at $\sim 100 \mathrm{GeV}$ (Albert et al. 2007), this emission should be easily detectable with $\sim 10^{2}-10^{5}$ photon counts. The emission from both close and distant encounters falls within the observed energy range and, under favorable conditions, the sensitivity of the LAT telescope on the GLAST satellite. With a collective area of $\sim 10^{4} \mathrm{~cm}^{2}$, LAT can detect $\sim 40$ photons from an on-axis red supergiant.

The GRB 940217 was one of the brightest ever detected. The EGRET telescope detected several $\sim 100 \mathrm{MeV}$ photons and one $18 \mathrm{GeV}$ photon $\sim 4500 \mathrm{~s}$ after the burst (Hurley et al. 1994). The energy of this mysterious late photon is compatible with an encounter of the blast wave with $\mathrm{O}$-star radiation at a distance of $\sim 1.5 \times 10^{17} \mathrm{~cm}$ (Fig. 2). The redshift of this burst is not known, but the implied fluence of high-energy afterglow photons, of the order $\sim 2 \times 10^{-3} \mathrm{~cm}^{-2}$ in the $\sim 10 \mathrm{GeV}$ range, is compatible with this scenario for a redshift of order unity. The detection of the scattered component in a burst of known redshift can strongly constrain the properties of the companion star and of the circumburst medium through Eqs. (3), (4), and (6).

\section{Discussion and conclusions}

I studied the emission from the interaction of the GRB blast wave with the dense photon field of an $\mathrm{O}$ star located at $R_{\mathrm{S}} \sim 1-$ $3 \times 10^{17} \mathrm{~cm}$ from the center of the explosion. Such distance is similar to the observed mean distance of $\mathrm{O}$ stars in the very dense stellar environments of massive clusters where a large fraction of the Wolf-Rayet stars reside.

The conclusion from this analysis is that inverse Compton scattering of the stellar photons by electrons accelerated in the shock leads to powerful $\mathrm{GeV}$ emission that appears from just minutes up to one day after the burst. This emission can be detected by Cherenkov telescopes and GLAST. Such detection can probe the properties of the blast wave and the nearby star. 
Attenuation of the produced $\gamma$-rays by interaction with the extragalactic background light (EBL) is expected to result to a highenergy cutoff of the $\gamma$-ray spectrum that will put constraints on the poorly known EBL.

The external Compton emission discussed here appears at higher energy than the synchrotron-self-Compton emission predicted by the standard afterglow model does. Assuming that a fraction $\epsilon_{B} \sim 10^{-3}$ of the energy released in the shock goes into magnetic fields $B^{\prime}$, the energy of the synchrotron photons in the frame comoving with the flow $E_{\mathrm{syn}}^{\prime}=3 \gamma_{\mathrm{e}}^{2} e B^{\prime} / 4 \pi m_{\mathrm{e}} c \simeq$ $4 \epsilon_{\mathrm{e},-1}^{2} \epsilon_{\mathrm{B},-3}^{1 / 2} E_{54}^{3 / 2} n_{2}^{-1} R_{17}^{-9 / 2} \mathrm{eV}$ is much lower than that of the stellar photons $E_{\mathrm{s}}^{\prime}=\Gamma e_{\mathrm{s}}=400 E_{54}^{3 / 2} e_{\mathrm{s}, 1} n_{2}^{-1} R_{17}^{-3 / 2} \mathrm{eV}$ for the distances of interest.

In this work, the simplification of a constant density circumburst medium is used, although the medium, a product of the collision of stellar winds, is much more complex. The wind interaction consists of regions of $r^{-2}$ density profiles close to the stars and regions of shocked winds (Stevens et al. 1992). A shock propagating in this environment may result in complex optical and X-ray afterglow lightcurves (Ramirez-Ruiz et al. 2001; Nakar \& Granot 2007) that can also probe such an interaction.

The conditions discussed here are different from interaction with photon fields closer in the center of explosion. These include the photon field in the progenitor's funnel that can seed the GRB emission (Lazzati et al. 2000) and the shock-breakout photon field that can power an X-ray $/ \gamma$-ray precursor to the main burst (MacFadyen et al. 2001; Ramirez-Ruiz et al. 2002; Waxman \& Meszaros 2003). Furthermore, if present, the photon field of a stellar companion, combined with prolonged central engine activity, can result in afterglow $\gamma$-ray emission (Ramirez-Ruiz 2004). These fields are located at distances
$R \lesssim 10^{13} \mathrm{~cm}$ from the center of the explosion, and they can limit the maximum Lorentz factor to which the flow is accelerated through the so-called "Compton drag" (e.g. Ghisellini et al. 2000).

Acknowledgements. I thank Henk Spruit for important suggestions and discussions and Jonathan Braithwaite for comments on the manuscript.

\section{References}

Albert, J., Aliu, E., Anderlub, H., et al. 2007, ApJ, 667, 358 Blandford, R. D., \& McKee, C. F. 1976, Phys. Fluids, 19, 1130 Blanch, O., \& Martinez, M. 2005, Astropart. Phys., 23, 588 Chevalier, R. A., \& Li, Z.-Y. 2000, ApJ, 536, 195

Crowther, P. A. 2007, ARA\&A, 45, 177

Figer, D. F. 2004, in The Formation and Evolution of Massive Young Star Clusters, ed. H. J. G. L. M. Lamers, L. J. Smith, \& A. Nota (San Francisco: ASP), ASP Conf. Ser., 322, 49

Galama, T. J., Vreeswijk, P. M., van Paradis, J., et al. 1998, Nature, 395, 670

Ghisellini, G., Lazzati, D., Celotti, A., \& Rees, M. J. 2000, MNRAS, 316, L45 Hjorth, J., Sollerman, J., Møoller, P., et al. 2003, Nature, 423, 847

Hurley, K., Matheson, T., Garnavich, P. M., et al. 1994, Nature, 372, 652

Lazzati, D., Ghisellini, G., Celotti, A., \& Rees, M. J. 2000, ApJ, 529, L17

MacFadyen, A. I., \& Woosley, S. E. 1999, ApJ, 524, 262

MacFadyen, A. I., Woosley, S. E., \& Heger, A. 2001, ApJ, 550, 410

Massey, P., \& Hunter, D. A. 1998, ApJ, 493, 180

Nakar, E., \& Granot, J. 2007, MNRAS, 380, 1744

Ramirez-Ruiz, E. 2004, MNRAS, 349, L38

Ramirez-Ruiz, E., Dray, L. M., Madau, P., \& Tout, C. A. 2001, MNRAS, 327, 829

Ramirez-Ruiz, E., MacFadyen, A. I., \& Lazzati, D. 2002, MNRAS, 331, 197

Sari, R., Piran, T., \& Narayan, R. 1998, ApJ, 497, 17

Stanek, K. Z., et al. 2003, ApJ, 591, 17

Stevens, I. R., Blondin, J. M., \& Pollock, A. M. T. 1992, ApJ, 386, 265

Waxman, E., \& Mészáros, P. 2003, ApJ, 584, 390

Wijers, R. A. M. J., \& Galama, T. J. 1999, ApJ, 523, 177 